\title{
Metamorphosis of the malaria parasite in the liver is associated with organelle clearance
}

\author{
Bamini Jayabalasingham ${ }^{1}$, Nazneen Bano ${ }^{1}$, Isabelle Coppens ${ }^{1}$ \\ ${ }^{I}$ Department of Molecular Microbiology and Immunology, Johns Hopkins Bloomberg School of Public Health, 615 N. Wolfe Street, \\ Baltimore, MD 21205, USA
}

Malaria parasites encounter diverse conditions as they cycle between their vertebrate host and mosquito vector. Within these distinct environments, the parasite undergoes drastic transformations, changing both its morphology and metabolism. Plasmodium species that infect mammals must first take up residence in the liver before initiating red blood cell infection. Following penetration into hepatocytes, the parasite converts from an invasion-competent, motile, elongated sporozoite to a metabolically active, round trophozoite. Relatively little is known about the cellular events involved in sporozoite metamorphosis. Our data uncover the early cellular events associated with these transformations. We illustrate that the beginning of metamorphosis is marked by the disruption of the membrane cytoskeleton beneath the plasma membrane, which results in a protruding area around the nucleus. As this bulbous region expands, the two distal ends of the sporozoite gradually retract and disappear, leading to cell sphericalization. This shape change is associated with major interior renovations and clearance of superfluous organelles, e.g. micronemes involved in invasion. The membrane cytoskeleton is reorganized into dense lamellar arrays within the cytoplasm and is partially expulsed by converting parasites. Simultaneously, micronemes are compartmentalized into large exocytic vesicles and are then discharged into the environment. At the completion of metamorphosis, the parasites only retain organelles necessary for replication. These observations lay the groundwork for further investigations on the developmental pathways implicated in the metamorphosis of the malaria parasite.

Keywords: malaria; hepatic stage; differentiation; membrane cytoskeleton; micronemes; exocytosis

Cell Research (2010) 20:1043-1059. doi:10.1038/cr.2010.88; published online 22 June 2010

\section{Introduction}

Malaria parasites must be transmitted from the mosquito vector to the mammalian host as part of their lifecycle. Upon entering the mammalian host, extracellular malaria sporozoites reach the liver and invade hepatocytes, wherein they undergo spectacular phenotypic changes prior to multiplication [1]. The mature merozoite form of the parasite that is released from hepatocytes is fully competent to subsequently infect red blood cells and instigate the pathology associated with malaria [2]. The process of recognition and entry into hepatocytes by sporozoites has been extensively studied but very little is

\footnotetext{
Correspondence: Isabelle Coppens

Tel: +1-443-287-1589; Fax: +1-410-955-0105

E-mail: icoppens@jhsph.edu

Received 11 December 2009; revised 18 March 2010; accepted 12 April

2010; published online 22 June 2010
}

known about the mechanisms involved in their structural differentiation into the replicative form.

The sporozoite is adeptly equipped for migration through tissues and invasion of mammalian hepatocytes. Extremely motile, elongated and finely curved, it possesses a robust membrane cytoskeleton essential for the maintenance of its shape and motility. Indeed, the sporozoite is delimited by a specialized cortical structure known as the trilaminar pellicle, which is composed of the plasma membrane tightly associated with flattened membrane cisternae forming the inner membrane complex (IMC) [3]. The IMC is continuous along the anterior two-thirds of the sporozoite. Further mechanical stability is provided to the pellicle by microtubules that are connected to the cytoplasmic face of the IMC by long linker molecules [4]. In addition to the IMC, the invasive form of the parasite has unique secretory organelles, e.g. micronemes and rhoptries that discharge their content at the time of host cell contact, facilitating productive 
invasion of the host cell. Micronemal proteins such as the thrombospondin-related anonymous protein (TRAP) contribute to hepatocyte adhesion $[5,6]$ while the content of the rhoptries is implicated the biogenesis of the parasitophorous vacuole (PV), a unique niche wherein the parasite multiplies in hepatocytes [7].

Enclosed in its PV, the sporozoite first displays an astonishing phase of metamorphosis associated with profound morphological modifications within the first $\sim 24 \mathrm{~h}$ post-invasion (p.i.) as it develops into a round trophozoite [8-12]. Subsequent to this metamorphosis, a mitotic phase begins, characterized by multiple fissions of parasite nuclei, transforming the parasite into a giant syncytium-like compartment. The replicative capacity of intrahepatic Plasmodium is remarkable as the parasite achieves one of the fastest growth rates among eukaryotic cells. To satisfy its nutritional needs, the parasite actively upregulates the expression of host genes involved in metabolite transport and anabolism $[13,14]$ and it transforms its PV into a highly permeable compartment accessible to small host molecules [15]. The end of the schizogony marks a phase of biogenesis of organelles that are critical for erythrocyte invasion and the production of fully formed merozoites ( $50 \mathrm{~h}$ to $55 \mathrm{~h}$ p.i.). During this phase, the plasma membrane of the schizonts is recycled to form the plasma membrane of individual merozoites [16]. The PV membrane is ruptured, which results in host cell degradation by parasite proteases. The merozoites are then released in the host cytoplasm and packed into vesicles (merosomes) for transport to the bloodstream.

We assume that parasite metamorphosis must be highly sophisticated as the sporozoite meets the challenge of becoming a replication-competent trophozoite. It has been shown that sporozoite micronemes and rhoptries are not maintained in mature trophozoites [8-10]. To date, details of the cellular events leading to organelle elimination during sporozoite conversion remain unexplored. In many biological systems, membrane-bound organelles can be cleared by expulsion into the environment, selective proteolysis by the ubiquitin-proteasome system (UPS) and/or gross degradation by macroautophagy [1719]. In order to decipher the developmental pathways that are implicated in the metamorphosis of the malaria parasite in the liver, we scrutinized the morphological changes occurring in converting parasites, with special focus on the fate of the IMC and micronemes. In this paper, we provide compelling evidence that converting sporozoites expulse the IMC and micronemes into the $\mathrm{PV}$, both of which being unnecessary for the trophozoite stage development. This process may serve as a rapid and efficient way of clearing the parasites of incomplete- ly degraded cellular constituents. More broadly, these observations will expand our knowledge on sporozoite biology and lay the groundwork for further studies on the spectacular metamorphosis of the malaria parasite in mammalian hosts. Current available drugs that target the liver stage are limited [20]. Disruption of the organelle removal systems will likely be detrimental to progeny development in the liver and may prevent the massive production of infective merozoites.

\section{Results}

The formation of a protrusion in the middle of the parasite marks the beginning of sporozoite differentiation into trophozoite

From about 4 h p.i. onwards, Plasmodium sporozoites undergo spectacular phenotypic changes in liver cells. Microscopic examination was performed to scrutinize the sequential developmental transformations of Plasmodium berghei sporozoites into trophozoites in vitro. During the remodeling processes, the ' $\mathrm{C}$ '- or ' $\mathrm{S}$ '-shaped sporozoite became progressively round (Figure 1A). The initiation of metamorphosis is marked by the appearance of a protruding area in the middle of the parasite, originating on either the outer convex or the concave edge. Sporozoites maintained in axenic conditions for $\sim 9 \mathrm{~h}$ also developed into early liver forms and were morphologically similar to their intracellular counterparts (Figure 1B) [12], suggesting that triggers for sporozoite differentiation are not host cell-dependent. The same basic steps of sporozoite remodeling were observed for Plasmodium yoelii (Supplementary information, Figure S1). In order to decipher the 3-D ultrastructural features of converting sporozoites, we capitalized on the fact that sporozoites can convert extracellularly to perform scanning electron microscopy (EM) imaging on axenic parasites. These studies revealed that the outer surface of the median bulbous area appeared wrinkled (Figure 1B), presumably as a result of local indentations of the plasma membrane. The diameter of the bulge was about 1.5 and $3 \mu \mathrm{m}$ at 4 and $8 \mathrm{~h}$, respectively. As the bulbous region of the parasite expanded, the two distal ends of the sporozoite gradually retracted and eventually disappeared (see arrows in Figure 1B). This results in the smoothening of the surface and complete sphericalization, without any change in cell surface.

Nucleus protrusion occurs as a result of a loss in the membrane cytoskeleton rigidity

To determine the fate of the membrane cytoskeleton during sporozoite conversion, we performed immunofluorescence assays using antibodies against the myosin A tail domain interacting protein (MTIP) [21] associated 
A

B
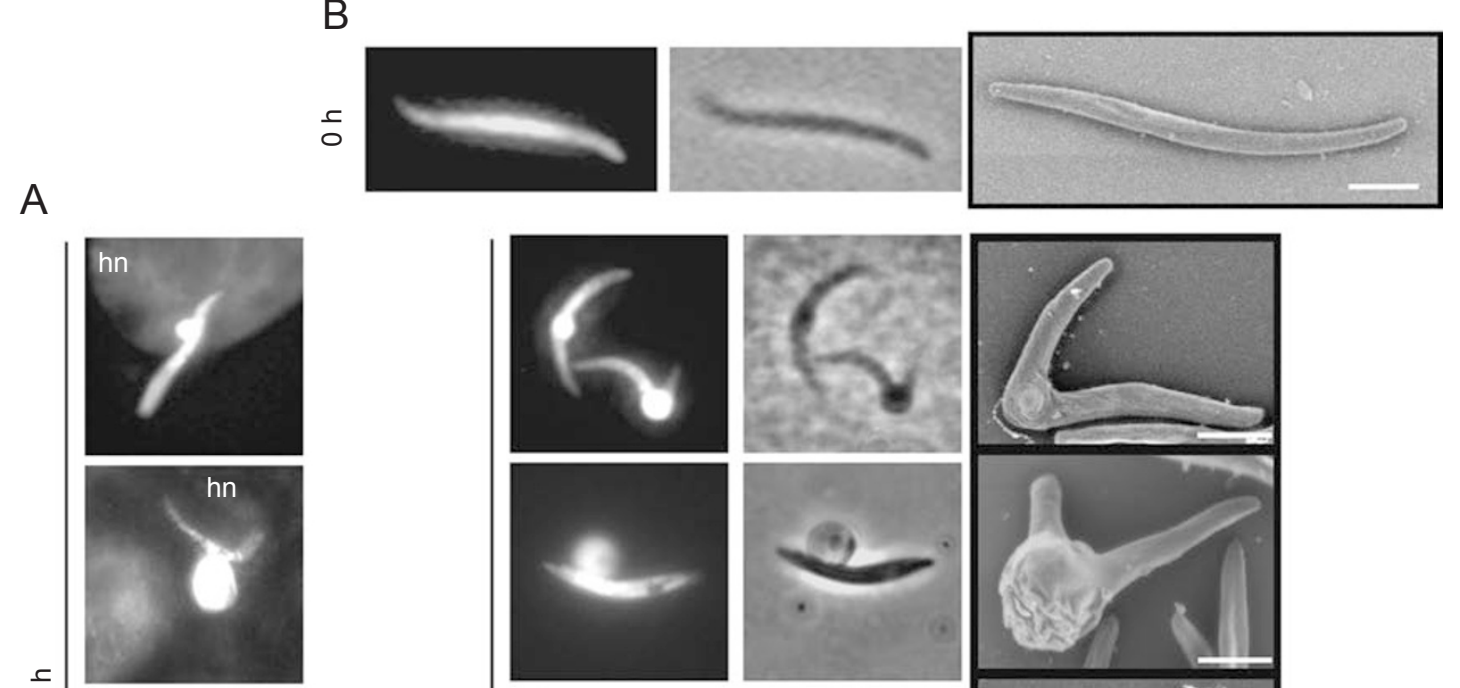

$\stackrel{\frac{1}{\varphi}}{\leftarrow}$
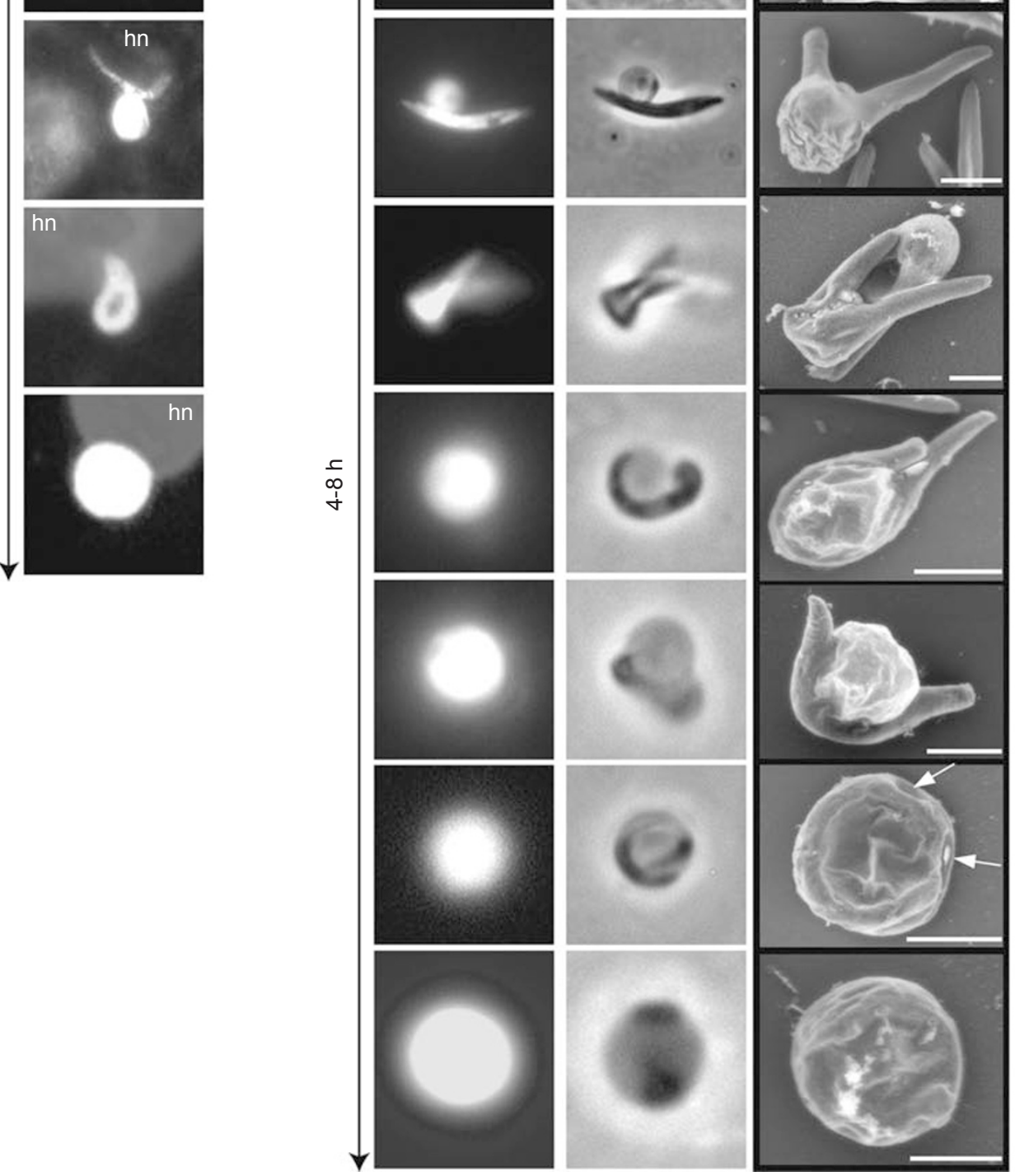

Figure 1 Morphological transformations of Plasmodium sporozoites into liver forms in vitro. (A) Fluorescence labeling of GFPexpressing $P$. berghei grown for 1 to $6 \mathrm{~h}$ in Hepa 1-6 cells showing parasite shape change. Nuclei are visualized by DAPI staining. hn, host nucleus. (B) Left: fluorescence microscopy and corresponding phase-contrast pictures of GFP-expressing $P$. berghei sporozoites immediately following isolation from salivary glands $(0 \mathrm{~h})$ or cultivated without host cells (axenically) for 4 to $8 \mathrm{~h}$, revealing parasite remodeling processes comparable to those observed for intracellular parasites. Right: scanning EM of untransformed $(0 \mathrm{~h})$ or converting $P$. berghei maintained axenically for 4 to $8 \mathrm{~h}$ showing the appearance of a median protruding area at the onset of parasite metamorphosis and progressive sphericalization. Note the simultaneous extension of the 'bulbous' portion and retraction of the two arm-like remnants of the sporozoite (arrows). Scale bars are $2 \mu \mathrm{m}$. 
with various proteins anchored to the IMC $[3,22]$. MTIP is part of the invasion machinery of the parasite. The presence of parasite Hsp70, which is highly expressed in liver stage parasites compared to sporozoites [23], was used as a marker for viability and stage conversion in our culture system. While the IMC was continuous along the length of the sporozoite, $7 \mathrm{~h}$ p.i., we consistently observed the disruption of the MTIP-labeled membranes at the central protruding area corresponding to the position of the nucleus (Figure 2A and 2B) as previously reported $[8,12,21]$. As the sporozoite rounds up, the IMC was found to retract along with the parasite extremities, and after complete transformation, MTIP staining appeared restricted to a small area at the parasite periphery.

The precise morphological changes occurring during sporozoite conversion into trophozoite were then inspected by EM on axenically cultured $P$. berghei and $P$. yoelii. In contrast to salivary gland sporozoites in which a tight connection is maintained between the plasma membrane and the IMC (labeled with anti-MTIP antibodies; Figure $3 \mathrm{~A}$ ), the continuity of the IMC was significantly impaired in converting parasites. Clearly points of IMC disruption were visible along the length of this organelle (Figure 3B, panels a and b) as evidenced by a lack of gold particles (against MTIP antibodies) under the plasma membrane (Figure 3B, panel c). This detachment of the IMC from the parasite surface resulted in local cytoplasm infiltration, inducing plasma membrane distention. The gradual dismantling of the IMC on both sides of the nucleus then provoked the protrusion of this organelle (Figure 3C). The loss of the IMC and microtubules from the nuclear area contributed to the wrinkled aspect of the surface membrane as observed by scanning EM (Figure $1 \mathrm{~B}$ and inset in Figure 3C, panel a). The intracellular dissociation of the IMC from the plasma membrane was concomitant to organelle redistribution in converting parasites (Supplementary information, Figure S2). The vast majority of secretory organelles were engulfed in the portion of the cytoplasm delineated by the retracting IMC (Figure 3C, panel b), while the nucleus, mitochondria and endoplasmic reticulum (ER) largely remained in the cytoplasmic area lacking the IMC. Some sections illustrated the dislocation of the anterior ring to the middle of the parasite (Supplementary information, Figure S3).

Following its dissociation from the plasma membrane at multiple foci (as seen in the serial sections depicted in Supplementary information, Figure S4A), the IMC is progressively compacted in the cytoplasm (Supplementary information, Figure S4B). Interestingly, membranous structures forming regular whorls with up to 10 layers were frequently observed in the cytoplasm next to the points of IMC breakdown (Figures 3C, panel c,
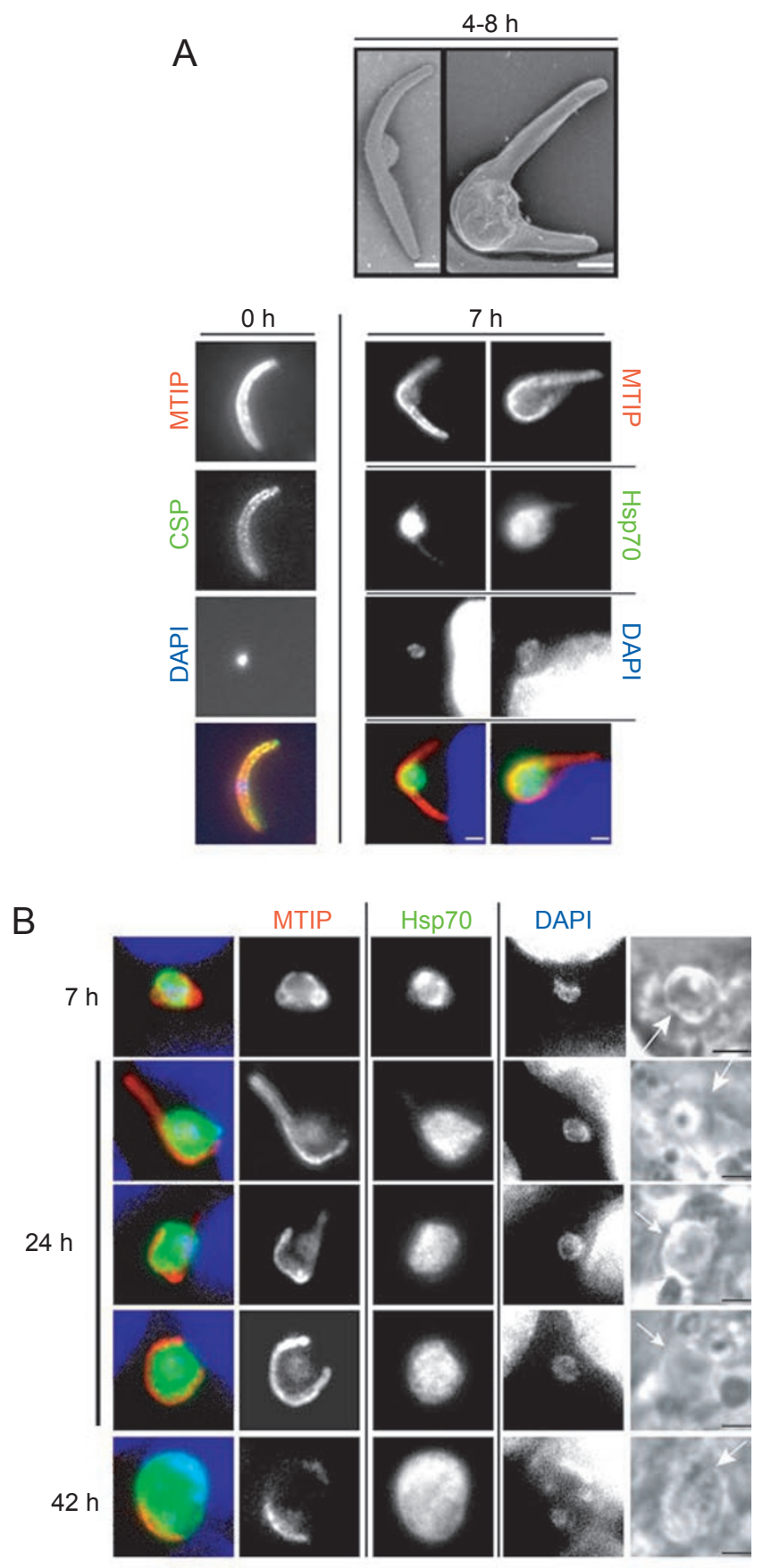

Figure 2 Changes in the morphology of the IMC during conversion. (A) Immunofluorescence assays of untransformed sporozoite $(0 \mathrm{~h})$ or intracellular $P$. berghei $(7 \mathrm{~h})$ labeled for the inner membrane complex (IMC) with anti-MTIP antibodies (red), the cytoplasm with anti-Hsp70 antibodies (green) and nuclear DNA with DAPI (blue), showing dismantling of the IMC in the perinuclear area. Images at the top correspond to scanning EM images of converting $P$. yoelii after $4 \mathrm{~h}$. Scale bars are $1 \mu \mathrm{m}$. (B) Immunofluorescence assays as described in (A) on intracellular $P$. berghei 7 to $24 \mathrm{~h}$, or $42 \mathrm{~h}$ p.i., illustrating the progressive decrease in IMC over the course of conversion. Scale bars are 2 $\mu \mathrm{m}$. 
A

a

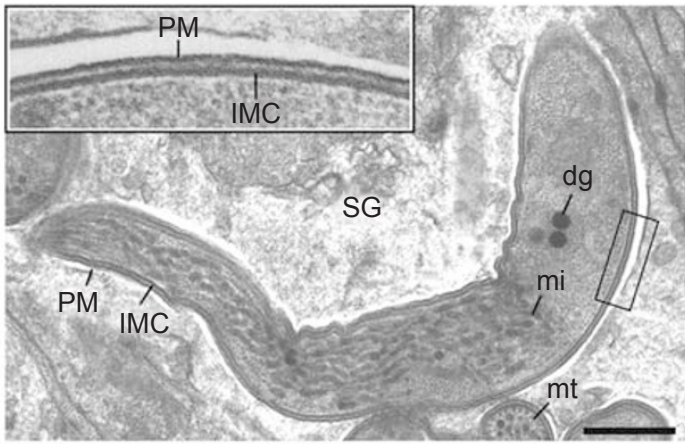

b

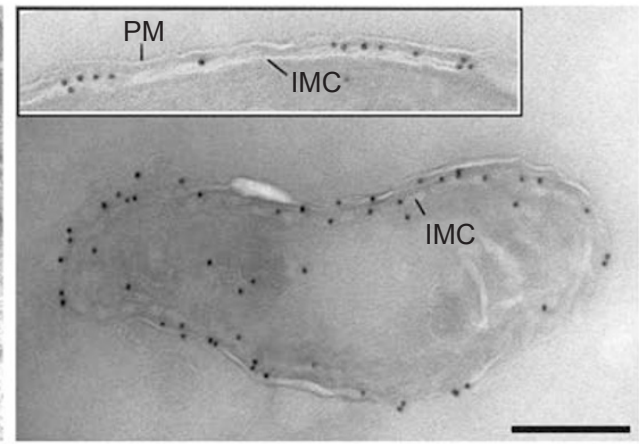

B

a

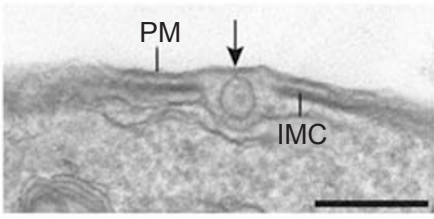

b

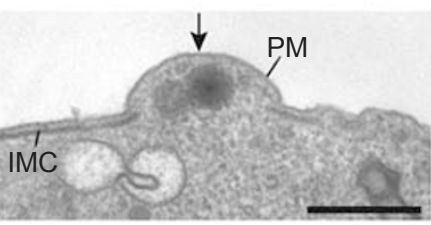

C

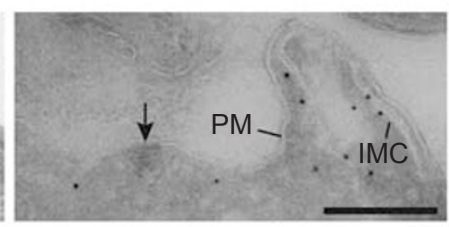

C

a

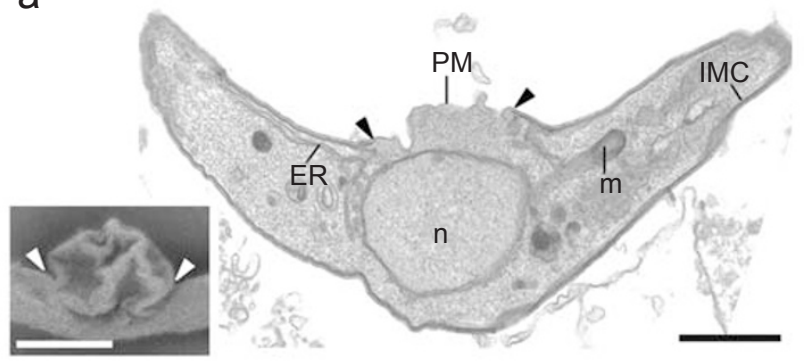

C

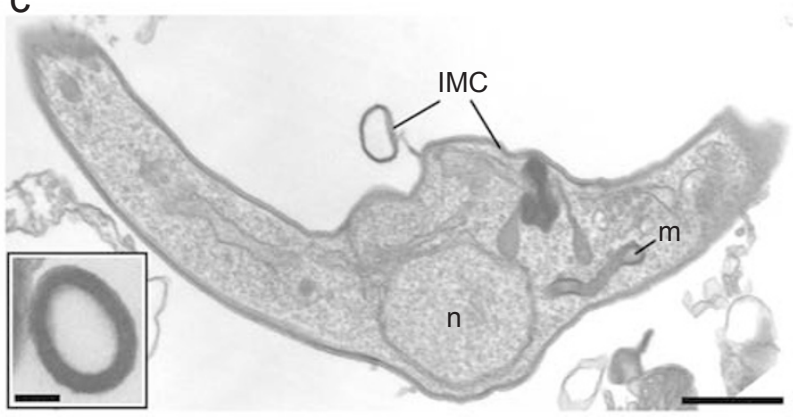

b

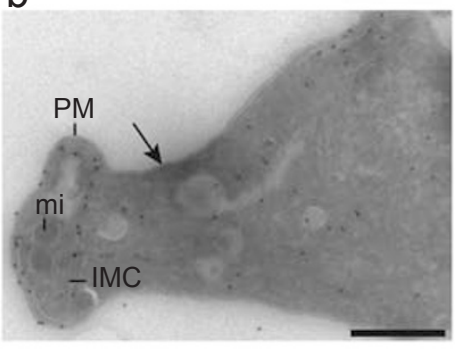

Figure 3 Ultrastructural evidence of IMC dismantling in converting parasites. (A) Transmission EM of $P$. berghei sporozoites in $A$. stephensi salivary glands illustrating the elongated cell shape during this stage and the presence of a continuous IMC on the intracellular face of the parasite as shown in the inset in panel $a$. Panel $b$ shows a cryosection of a sporozoite immunolabeled with anti-MTIP antibodies, revealed with protein A-gold particles. The anti-MTIP antibody staining is largely confined to the subpellicular IMC (inset). (B, C) Transmission and scanning EM of $P$. berghei maintained axenically for $4 \mathrm{~h}$. Arrows in (B) pinpoint areas of the plasma membrane lacking the IMC and associated with cytoplasm overgrowth. The cryosection of the parasite labeled with anti-MTIP antibodies in panel c in (B) confirms the dissociation of the IMC from the plasma membrane during conversion. Arrowheads in panel a in (C) show the disruption of the IMC on both sides of the nucleus. The scanning EM image in the inset reveals the ruffled aspect of the plasma membrane upon detachment from the IMC in the nuclear region. Panel $b$ in (C) is a cryosection of a parasite labeled for MTIP showing the retraction of the IMC to parasite extremities containing micronemes. Panel $\mathrm{c}$ in $(\mathrm{C})$ shows another section of the parasite seen in panel $a$, and the inset illustrates extracellular whorls. dg, dense granules; mi, micronemes; mt, microtubules; m, mitochondrion; $\mathrm{n}$, nucleus; PM, plasma membrane; SG, salivary glands. Scale bars are $0.5 \mu \mathrm{m}$ except inset in panel c in (C) $(0.1 \mu \mathrm{m})$. 
A
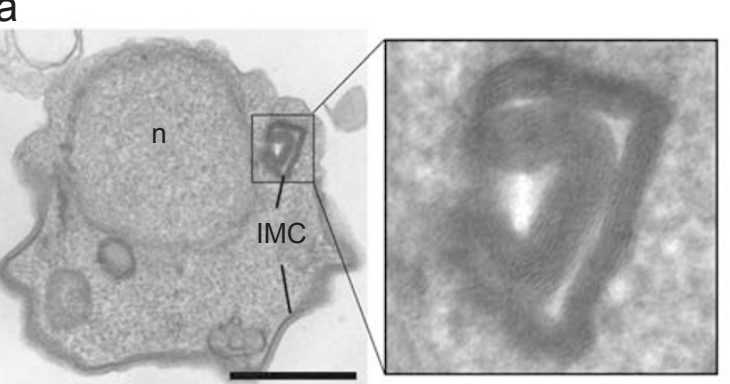

b

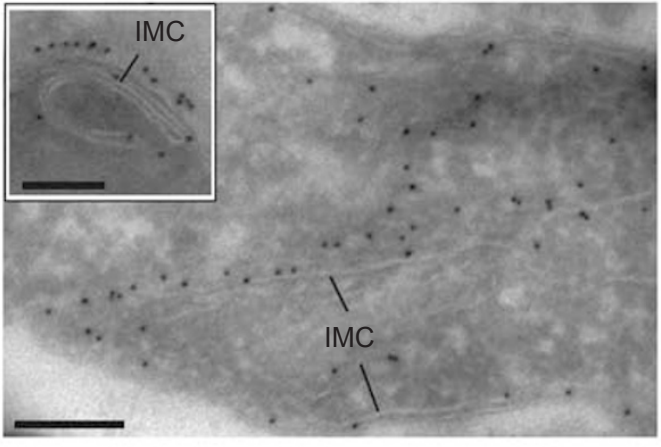

B

a
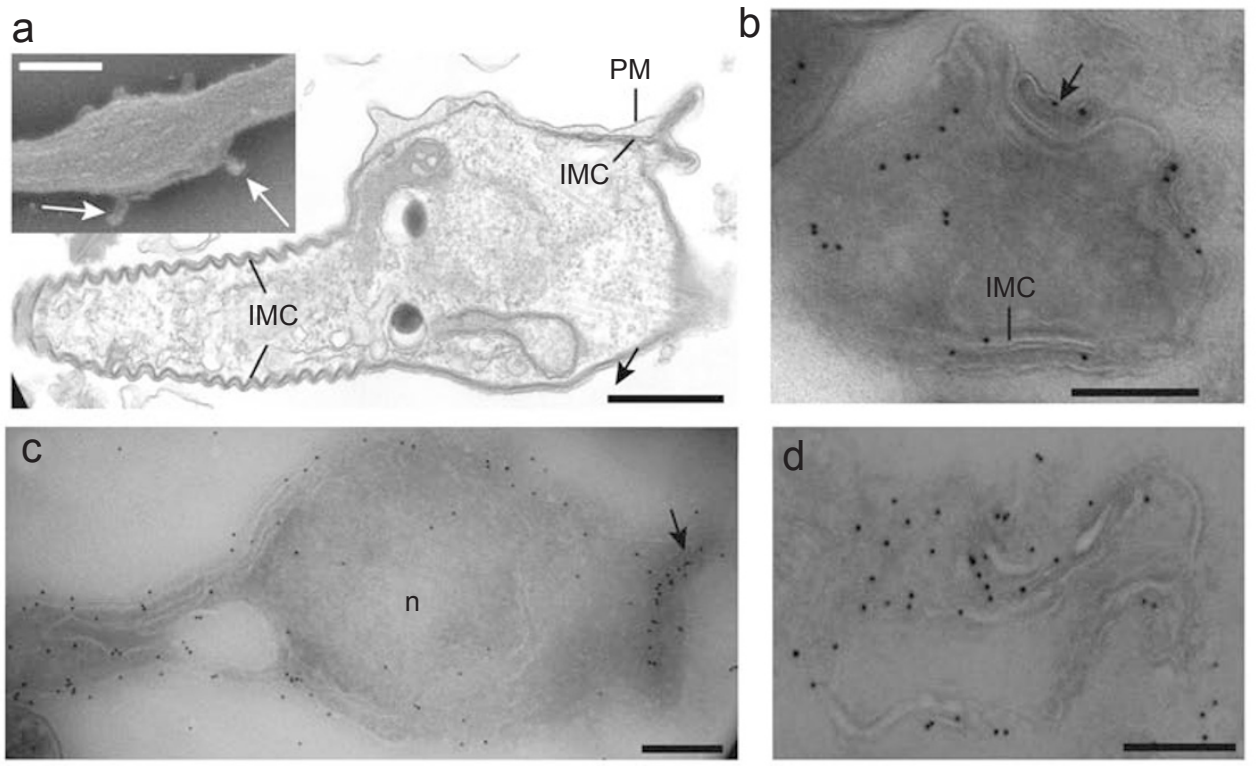

C

a b
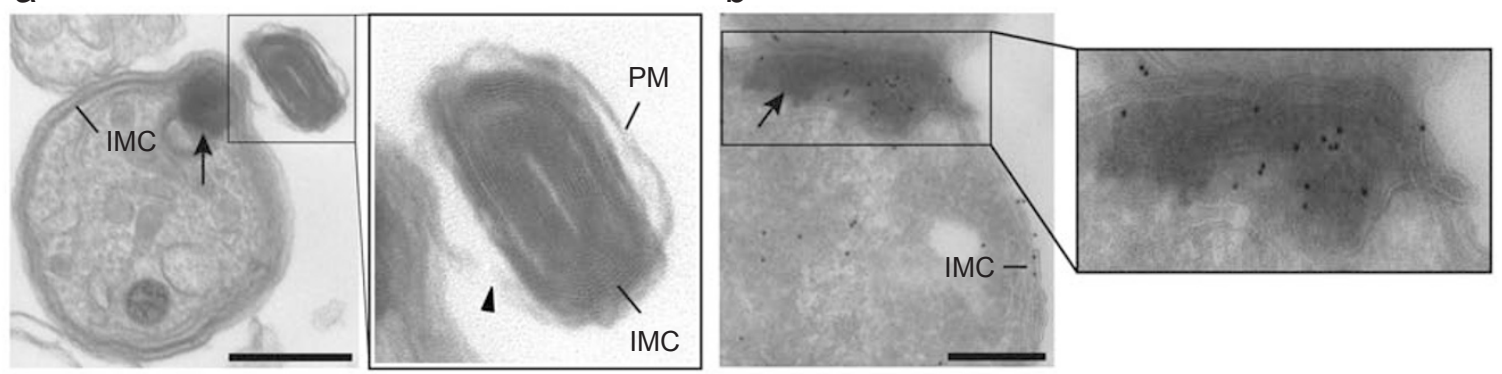

Figure 4 Morphological evidence of IMC packaging and expulsion from converting parasites. (A-C) Transmission and scanning EM of $P$. berghei (A, B) and $P$. yoelii (C) maintained axenically for $4 \mathrm{~h}$. The presence of profiles of circular IMC whorls are seen in parasites in (A) panel a. Panel $b$ and the inset are cryosections of parasite interior labeled for MTIP and show rearrangement of the IMC into circular folds in the cytoplasm. In (B), packaged membranes corresponding to the IMC are found appended to the parasite. IMC compaction is visible on the surface of the parasite in panel a and scanning EM imaging confirms the presence of bulges at the parasite surface (arrowheads in the inset). Cryosections of parasite immunolabeled for MTIP in panels $b$ and $c$ show corresponding pictures with MTIP accumulation at the edge of the sporozoite (arrows). The cryosection in panel d illustrate free membranous whorls positive for MTIP observed between parasites (panel d). (C) shows parasites with membranous whorls, presumably derived from the IMC and compacted in the cytoplasm before protrusion and release into the environment. The arrowhead pinpoints the plasma membrane surrounding the IMC. Panel b is a cryosection of MTIP-labeled membranes revealing the presence of electron-dense areas containing MTIP-labeled membranes (arrows) at the parasite periphery (b). n, nucleus; PM, plasma membrane. Scale bars are $0.5 \mu \mathrm{m}$. 
Figure 4A and Supplementary information, Figure S4C). Immunogold staining of parasites with anti-MTIP antibodies confirmed that the membranous whorls within the parasite were derived from the IMC (Figure 4A, panel b). Similar packaged membranes consisting of membranous sheaths surrounded by the plasma membrane were also observed externally, either budding from the parasite or appended to the surface (Figure 4B and 4C and Supplementary information, Figure S4D) as confirmed with MTIP staining (panels $b$ and $\mathrm{c}$ in Figure 4B and 4C). Free MTIP-labeled membranes were also detected between parasites (Figure 4B, panel d), suggesting that residual IMC is eventually expelled by the parasite. Therefore, it is tempting to propose that the reduction of mechanical constraints due to the removal of the IMC together with the loss of polarity of the parasite may lead to the round shape of the trophozoite form.

\section{Sporozoite development progresses more rapidly within a $P V$ than axenically between cells}

We then examined the peculiarities of $P$. yoelii sporozoite differentiation in intracellular conditions. After invasion, $P$. yoelii was most commonly found to lie in a narrow PV (Figure 5A), preferentially located in the host perinuclear area [15] (Figure 1A). Within $1 \mathrm{~h}$ p.i., the PVM showed close association with host rough ER (Figure 5B). Host ribosomes were restricted to the face of the ER not associated with the PV membrane. The identity of intracellular parasites at early time points p.i. was confirmed by their elongated crescent shape and the presence of (i) the IMC beneath the plasma membrane, (ii) subpellicular microtubules and (iii) secretory organelles. Major changes in these three structures and composition were visible in parasites $4 \mathrm{~h}$ p.i. As observed for axenic parasites, the area of the parasite containing the nucleus was enlarged and round (Figure 5C) and in some regions, the IMC was detached, leaving the plasma membrane bare (Figure 5D). Secretory organelles were encased in the extremities still delineated by the IMC. By $12 \mathrm{~h}$ p.i., the nuclear area of the parasites enlarged further, concomitant with a reduction in length of the distal ends. These 12-h forms were characterized by the presence of several membrane whorls in the cytoplasm (Figure $5 \mathrm{E}$ and $5 \mathrm{~F}$ and Supplementary information, Figure S4) and the accumulation of osmiophilic material in the PV (Figure 5F and 5G and Supplementary information, Figure S5), e.g. packaged membranes likely derived from the IMC. By comparison, the 12-h forms that developed intracellularly appeared larger and rounder than those maintained in axenic conditions. Similarly, parasites that did not invade cells but instead developed extracellularly between hepatic cells for $12 \mathrm{~h}$ underwent early meta- morphosis but exhibited a delayed conversion, as they displayed morphological features closer to those of the intracellular 4-h forms (data not shown). These results underline the substantial contribution of host cell factors to optimal sporozoite conversion.

At the completion of metamorphosis, the parasites only retain organelles involved in biosyntheses

During the process of conversion, the PV membrane also underwent dynamic transformations to adapt to the shape changes of the sporozoite (Figure 5H). Astonishingly, host ER-PV interaction was constantly maintained over the course of metamorphosis, indicative of strong association between the vacuolar membrane and the host ER network (Figures 5 and 6).

By $24 \mathrm{~h}$ p.i., the parasites increased in volume by 4 to 5 times compared to sporozoites and intrahepatic $12 \mathrm{~h}$ forms, both of which have comparable cell volumes $(\sim 35$ $\left.\mu \mathrm{m}^{3}\right)$. The 24-h forms still retained some portion of the IMC (Figure 6A-6D). 3-D visualization of MTIP-labeled membranes at this stage using spinning disk confocal microscopy illustrated the persistence of residual IMC, as a retracting network within the parasite, confined in part to the parasite periphery (Figure 6E; see Supplementary information, Video S1). By contrast, the anterior ring and secretory organelles involved in invasion were absent in all the sections viewed. The cytoplasm, which had a high electron density in sporozoites due to abundant ribosomes, now appeared less electron-dense with scattered ribosomes. The nucleus became enlarged and lobed, which is indicative of mitotic events occurring within the intact nuclear envelope (Figure 6A). At this stage of development, the 24-h forms - or trophozoites - showed no resemblance to sporozoites. The nucleoplasm exhibited the same texture as the cytoplasm (Figure 6A-6D). The general appearance of the ER and mitochondrion was also noticeably different in trophozoites (Figure 6F and $6 \mathrm{G}$ ) compared to sporozoites (Figure $6 \mathrm{H}$ ). The ER expanded markedly and reorganized into several local dense networks with shorter sheets and dilated cisternae, resembling mitotic ER in mammalian cells [24]. The single mitochondrion of the sporozoite developed into a major network with numerous small branches, suggesting major restructuring of this organelle as visualized by staining with mito-Tracker (Figure 6I). The parasite also retained the apicoplast, a relict plastid that harbors essential anabolic pathways [25], as shown by immunofluorescence using antibodies against the apicoplast resident acyl-carrier binding protein (ACP). Like the mitochondrion, the apicoplast develops from a simple structure to a highly branched continuous network during schizogony [26]. 

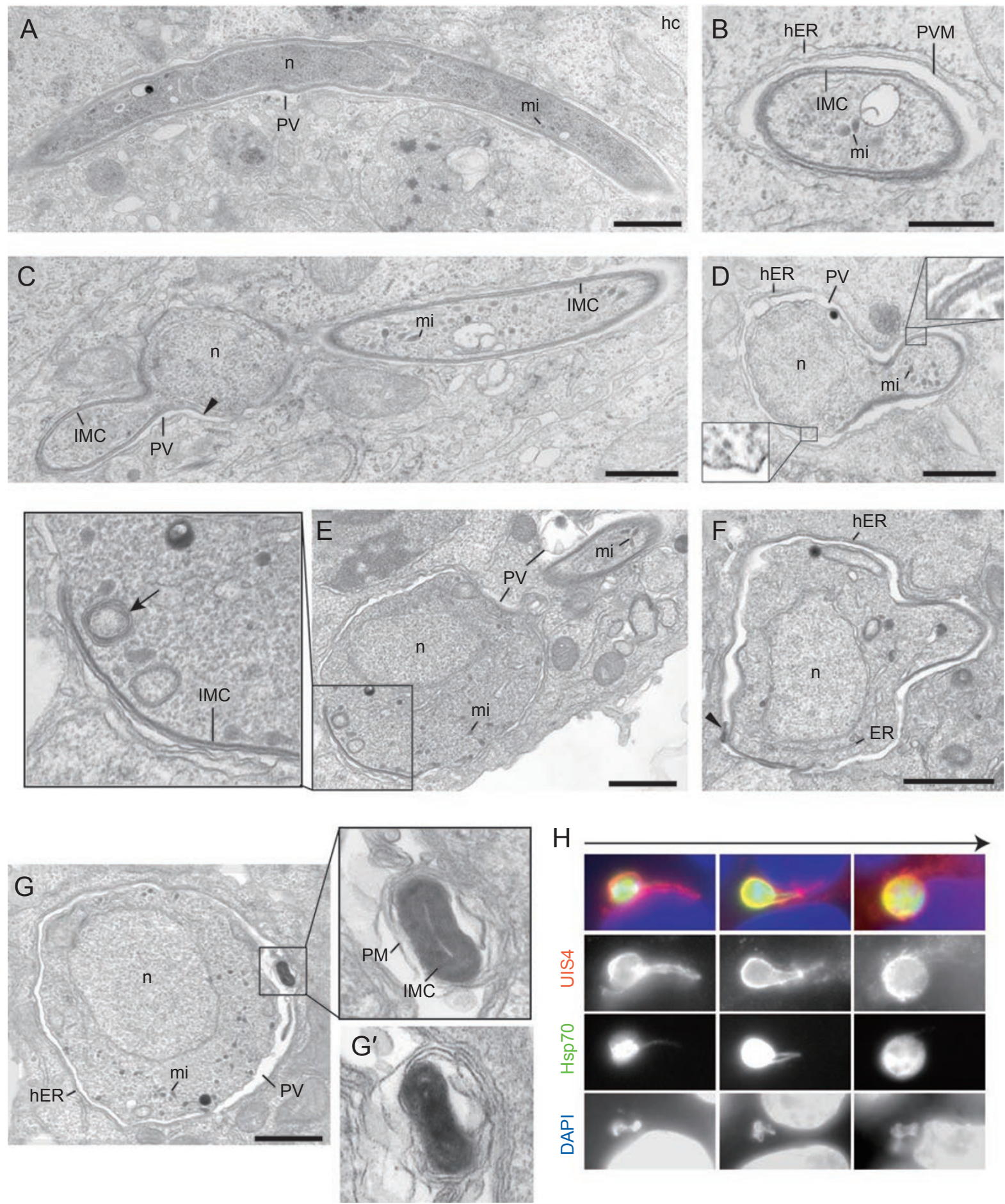

Figure 5 Morphological features of early conversion of sporozoites within the PV. (A-G) Transmission EM of $P$. yoelii-infected HepG2-CD81 cells $1 \mathrm{~h} \mathrm{(A,} \mathrm{B),} 4 \mathrm{~h}$ (C, D) or $12 \mathrm{~h}$ p.i. (E, F). After invasion, the parasite resides in a narrow PV that closely associates with host ER (hER). The arrowhead in (C) pinpoints a part of the pellicle (IMC and plasma membrane) in the protruding nuclear area (n) in which the IMC is detached from the plasma membrane (PM). Insets in (D) illustrate part of the plasma membrane delineated by the IMC (upper) or stripped of the IMC (lower) for direct comparison. Arrows in (E) show cytoplasmic membranous whorls underneath the pellicle. Packaged membranes in the PV lumen are pinpointed by the arrowhead in (F) and magnification of (G-G') (serial sections). mi, micronemes. Scale bars are $1 \mu \mathrm{m}$. (H) Immunofluorescence assays of transforming P. yoelii $24 \mathrm{~h}$ p.i. in HepG2-CD81 cells labeled with anti-Hsp70 antibodies (green), anti-UIS4 (red) to visualize the PVM and DAPI (blue), showing conformation changes of the PVM as the parasite rounds up. 

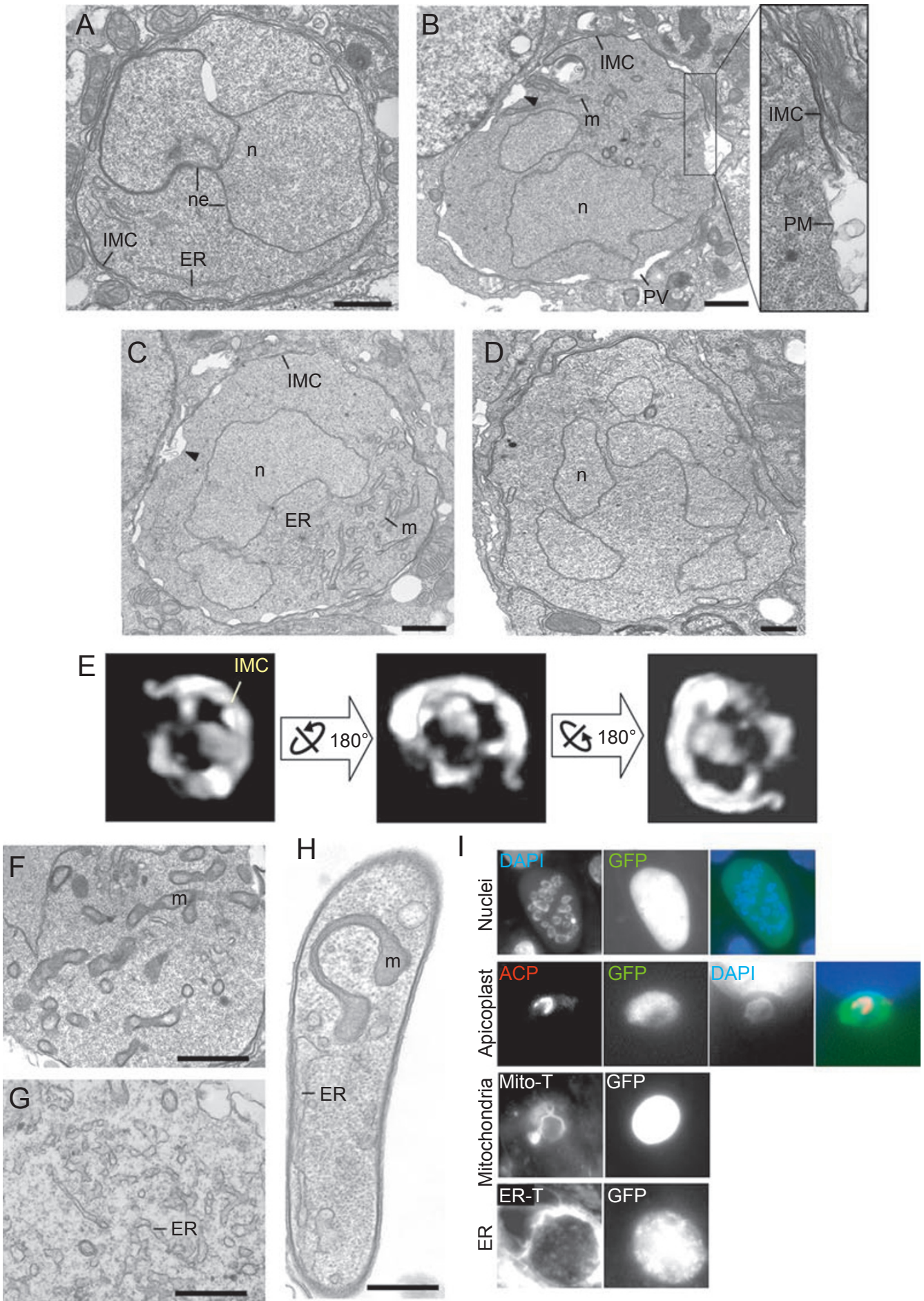

Figure 6 Organelle composition in replicating liver forms at the onset of karyokinesis. (A-D) Transmission EM of $P$. yoeliiinfected HepG2-CD81 cells $24 \mathrm{~h}$ p.i. showing endomitotic profiles and residual IMC. Arrowheads pinpoint some areas lacking IMC. n, nucleus; ne, nuclear envelope. Scale bars are $1 \mu \mathrm{m}$. (E) Spinning disk confocal microscopy of intracellular $P$. berghei for $24 \mathrm{~h}$ before labeling with anti-MTIP antibodies showing 3-D reconstruction of the IMC. On all viewed angles the IMC appears as a condensed network. (F, G) Transmission EM of $P$. yoelii-infected HepG2-CD81 cells $24 \mathrm{~h}$ p.i. showing large areas occupied by ER and mitochondria $(\mathrm{m})$. Scale bars are $1 \mu \mathrm{m}$. $(\mathbf{H})$ Transmission EM of $P$. yoelii sporozoites showing the mitochondrion and ER for comparison with these organelles in parasites shown in (F and $\mathbf{G})$. Scale bars are $1 \mu \mathrm{m}$. (I) Fluorescence labeling of GFP-expressing $P$. berghei labeled with DAPI and anti-ACP antibodies to identify the apicoplast, MitoTracker (Mito-T) or ER-Tracker to visualize these organelles. 
It has been previously observed that sporozoites occasionally penetrate into the host nucleus but these intranuclear forms display a limited degree of maturation [27]. We also detected P. yoelii in the host nucleoplasm. These few intranuclear parasites were never surrounded by any membrane (Supplementary information, Figure S6). Within $24 \mathrm{~h}$, only a small number of these parasites completed metamorphosis unlike their intravacuolar counterparts. In addition, the intranuclear parasites never underwent endomitoses. A decrease in parasite viability was similarly observed for sporozoites cultivated under axenic conditions for $24 \mathrm{~h}$ as no nuclear divisions were observed in extracellular trophozoites but instead parasites became progressively vacuolized (data not shown).
This indicates that the PV represents a suitable environment that is favorable for sporozoite conversion and essential for schizogony.

Micronemes are compartmentalized in the cytoplasm prior to their discharge in the parasitophorous vacuole

Sporozoite micronemes are small, oval structures with a diameter of $\sim 50 \mathrm{~nm}$. These organelles are abundantly distributed throughout the cytoplasm of sporozoites as shown by the diffuse pattern observed by fluorescence microscopy on parasites labeled with anti-TRAP antibodies (Figure 7) $[28,29]$. ImmunoEM staining of sporozoites with the same antibodies confirmed the abundance of micronemes dispersed in the cytoplasm (see Figure 9A).
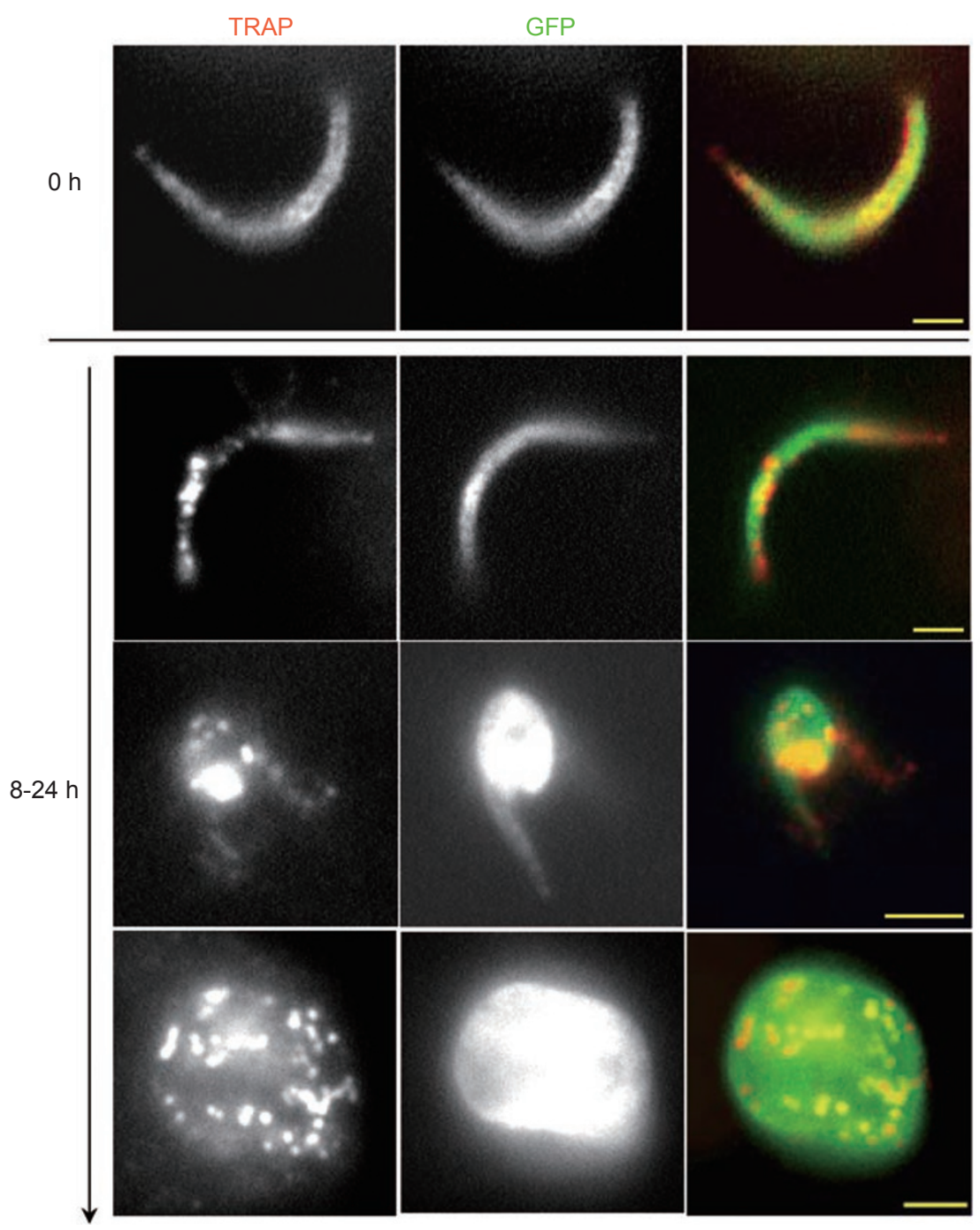

Figure 7 Compartmentalization of micronemes during conversion. Immunofluorescence assays on GFP-expressing $P$. berghei sporozoite $(0 \mathrm{~h}$ ) or Hepa 1-6 cells infected with GFP-expressing P. berghei ( 8 and $24 \mathrm{~h}$ p.i.) labeled with anti-TRAP antibodies (red) to detect micronemes. During conversion, the diffuse staining of micronemes in sporozoites becomes punctate. Scale bars are $2 \mu \mathrm{m}$. 
We monitored the localization of TRAP by fluorescence microscopy to follow the kinetics of microneme removal over the time. Surprisingly, during the process of parasite transformation, large and well-defined punctate structures containing TRAP were visible. The diameter of the TRAP-containing organelles varied from 0.2 to $0.4 \mu \mathrm{m}$, and likely reflected either an aggregation of micronemes in the cytoplasm or their compartmentalization within a membrane-bound structure. 8 and 24 h p.i., the TRAPcontaining organelles were distributed randomly in the cytoplasm (Figure 7). By 31 h p.i., these compartments became noticeably smaller and were more abundantly concentrated at the periphery of the cytoplasm (Figure $8 \mathrm{~A})$. On late schizonts, the fluorescence staining was predominantly at the outer surface of the parasite, likely in the vacuolar space, suggesting a discharge of TRAP into
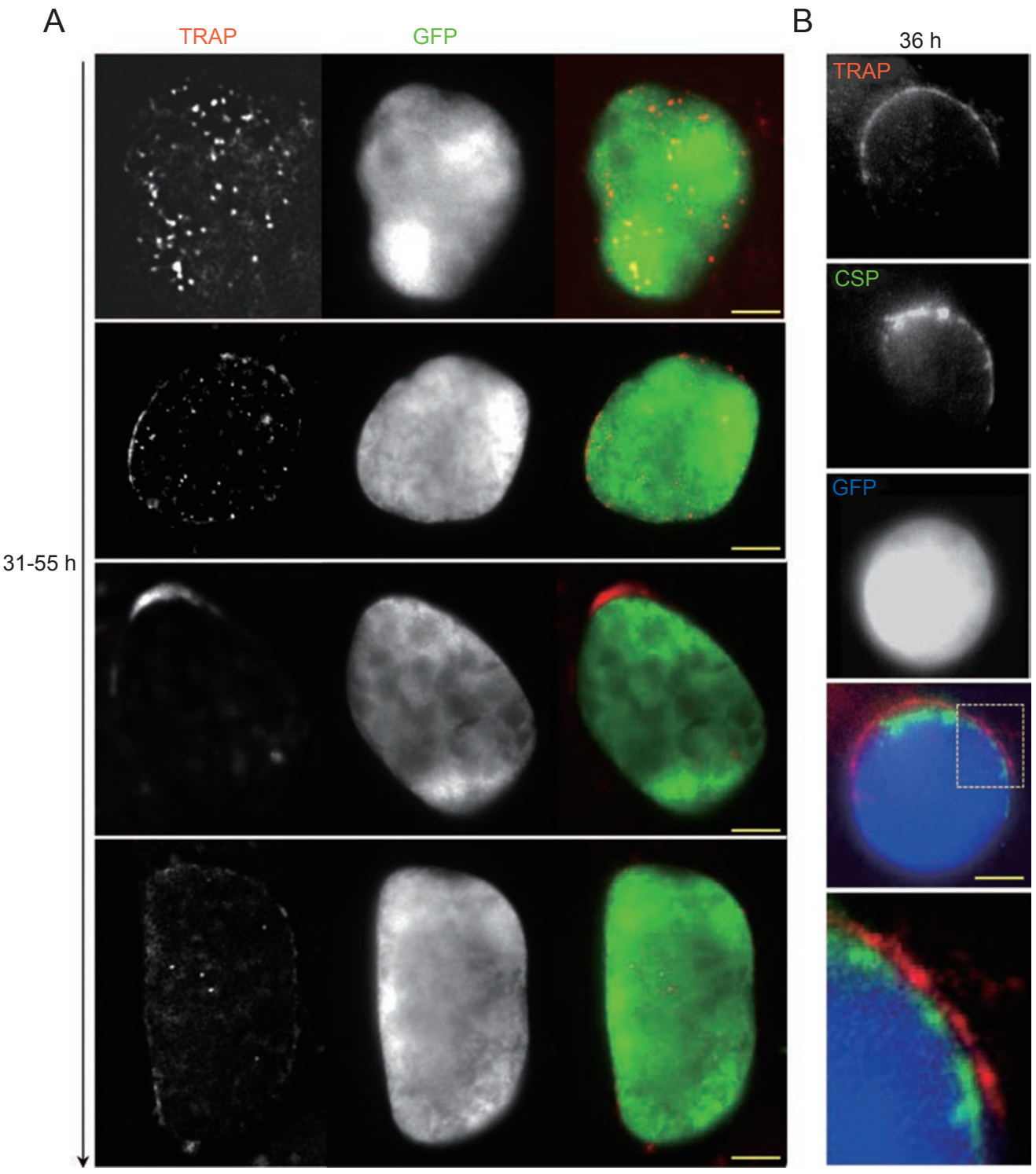

Figure 8 Elimination of micronemes during conversion. (A) Immunofluorescence assays on Hepa 1-6 cells infected with GFP-expressing $P$. berghei $(31,43,55$ h p.i.) labeled with anti-TRAP antibodies (red) to detect micronemes. (B) Immunofluorescence assays on Hepa 1-6 cells infected with GFP-expressing P. berghei (36 h p.i.) labeled with anti-TRAP (red) and antiCSP (green) antibodies for the plasma membrane. Note that the CSP labeling is not continuous along the parasite plasma membrane due to an arrest of translation of CSP by the parasite $24 \mathrm{~h}$ after hepatocyte invasion. GFP pattern is pseudocolorized in blue. At the end of conversion, the staining of micronemes in schizonts is eventually confined to the periphery of the parasite, beyond the CSP staining, indicative of a release of TRAP into the vacuolar space. Scale bars are $2 \mu \mathrm{m}$. 
A
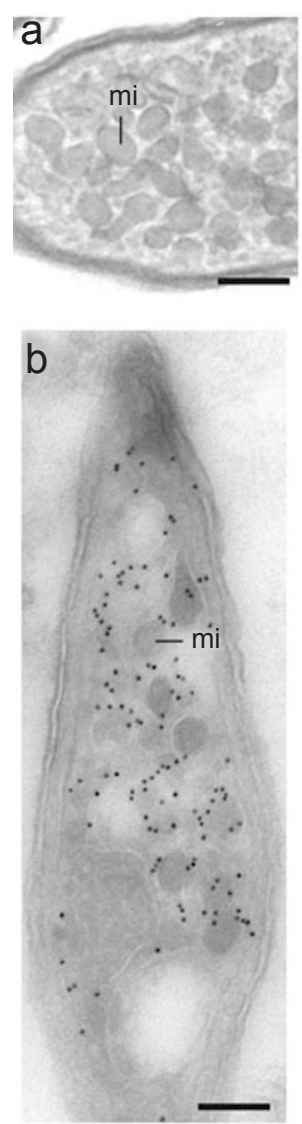

B
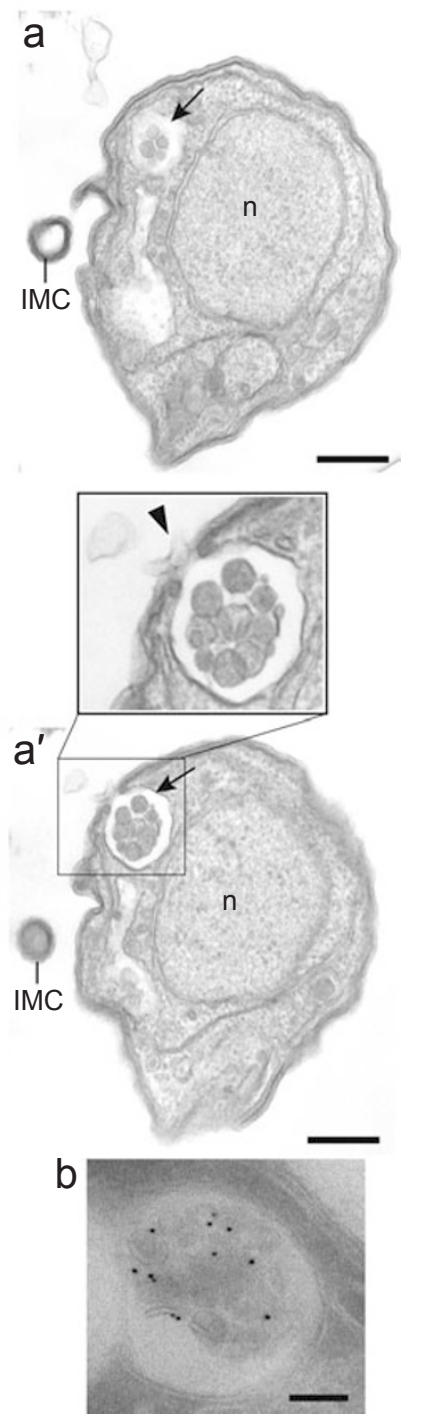

Figure 9 Ultrastructural evidence of microneme compartmentalization during conversion. (A) P. yoelii sporozoites observed by transmission EM (a) or after immunolabeling with anti-TRAP antibodies (b) confirming microneme ( $\mathrm{mi}$ ) distribution throughout the cytoplasm of unconverted parasites. (B) Transmission EM of axenic $P$. yoelii incubated for $8 \mathrm{~h}$ (serial sections in panels a-a') showing sequestration of micronemes in a single membranebound compartment (B, arrows). The arrowhead in (B) pinpoints the opening of the compartment to the exterior. The identity of micronemes has been confirmed by immunoEM with anti-TRAP antibodies (b in B). Scale bars are $0.1 \mu \mathrm{m}$.

the PV or the expulsion of intact TRAP-containing organelles by the parasite. Double immunostaining of parasites $36 \mathrm{~h}$ p.i. with anti-TRAP and anti-CSP antibodies confirmed that TRAP staining was outside of the parasite plasma membrane (Figure 8B). The majority of the 55-h forms did not show any TRAP labeling in their PV, im-

plying mechanisms of organelle degradation operational in the vacuolar space.

To further characterize these large TRAP-positive structures, we scrutinized the parasites at the ultrastructural level. EM studies on parasites $8 \mathrm{~h}$ post-conversion revealed the presence of $\sim 0.25 \mu \mathrm{m}$ organelles delineated by a single membrane and containing intraluminal vesicles having the same size and electron density as sporozoite micronemes (Figure 9B, compared with Figure 9A, panel a). ImmunoEM labeling demonstrated the presence of TRAP on vesicles sequestered within single membrane-bound compartments (Figure 9B, panel b), which confirms that these structures are indeed micronemes. Interestingly, some microneme-containing compartments were docked onto the plasma membrane at sites devoid of IMC. This suggests the occurrence of fusion events permitting the exocytosis of micronemes into the PV (see serial sections a-a' in Figure 9B).

\section{Discussion}

Plasmodium parasites adopt two conformations throughout the asexual phase of their lifecycle: a motile, invasive form and a replication-competent metabolically active form [30]. Conversion between these two forms requires the parasite to drastically change its morphology and metabolism. In hepatocytes, the motile sporozoite must convert into an intracellular form suitable for replication to generate thousands of infective merozoites within 3-7 days, depending on the Plasmodium species [11]. In this paper, we have explored the cellular remodeling processes that occur during the metamorphosis of sporozoites. Based on our observations, we propose a model in which the sporozoite undergoes a coordinated series of morphological changes accompanied by organelle clearance in order to transform into the liver stage trophozoite (Figure 10). The initial stages of sporozoite transformation are grossly comparable to the transformation of the Plasmodium ookinete to oocyst in the mosquito, except that it takes only $24 \mathrm{~h}$ for the sporozoite, compared to the 8 days required for the formation of young oocysts [31]. Upon completion of this conversion, the mature trophozoite largely lacks the cytoskeletal architecture that was providing mechanical support to the sporozoite plasma membrane and would have been incompatible with cytoplasm overgrowth. Intriguingly, disruption of the IMC was consistently observed to originate near the nucleus. A similar hump was visible near the centrally located nucleus of $P$. berghei ookinetes, during their transformation into oocysts in the mosquito's midgut, and this phenotype may also be correlated with the breakdown of the IMC in this location [31]. Recent 


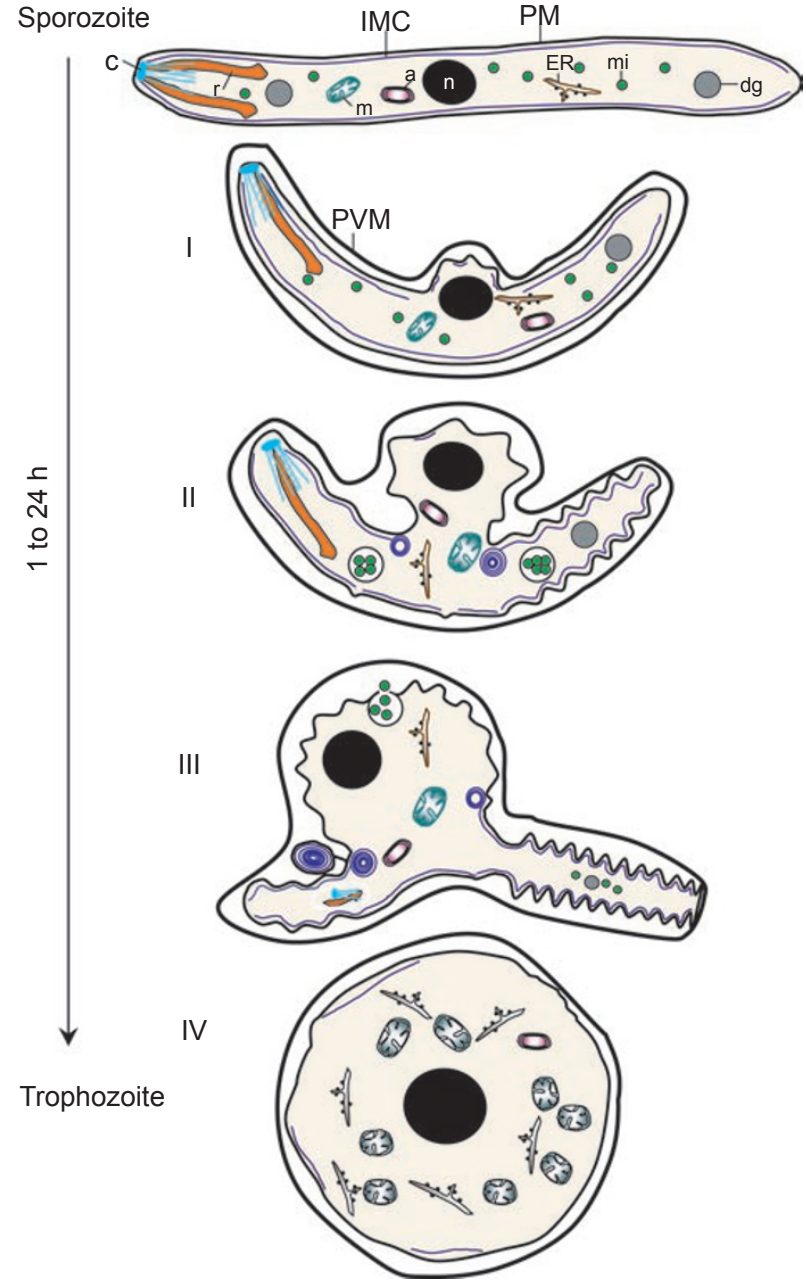

Figure 10 Hypothetical model of the remodeling processes of Plasmodium sporozoites in liver cells. Sporozoite metamorphosis into liver forms begins with local disruption of the IMC leading to cytoplasm expansion (stage I). Superfluous organelles such as micronemes are then compartmentalized while the IMC is packaged forming compact whorls (stage II). The micronemecontaining compartments and coiled IMC are discharged in the vacuolar space, indicative of exocytosis (stage III). At the end of the metamorphosis, a minimal set of organelles required to sustain rapid growth is retained in the cytoplasm (stage IV or trophozoite), and 24-h p.i. nuclear divisions begin (schizont stage). a, apicoplast; dg, dense granules; mi, micronemes; n, nucleus; $\mathrm{PM}$, plasma membrane.

tomography studies revealed that the nuclear envelope membranes of sporozoites are associated closely with the IMC at one side of the parasite with connectors of $\sim 25$ $\mathrm{nm}$ length [4]. In the areas of physical contact with the IMC, the two leaflets of the nuclear envelope are closely apposed to each other and no nuclear pores were detected. These observations suggest a coordinated movement between the IMC and nucleus. In the future, it would be interesting to examine whether IMC disruption preferentially occurs at the side of the nucleus that is connected to the IMC and what could be the potential role of these linkers during the morphological transformations of the parasite. Relevantly to this, sporozoites lacking IMC1a, a cytoskeletal protein essential for its mechanical stability, have a weakened cell structure [32]. This is manifested by a protruding area around the nucleus that closely resembles that of sporozoites converting into liver forms. It is therefore plausible that proteins such as PbIMC1a are degraded during sporozoite conversion to facilitate parasite sphericalization. Sporozoite organelles specialized for host cell invasion and thus not longer needed at this stage are also missing from fully converted trophozoites. Only a set of biosynthetic organelles implicated in nucleic acid, protein and lipid production are retained and become markedly expanded in these actively growing forms. They include the ER, mitochondrion, nucleus and apicoplast.

We observe that all viable sporozoites dismantle their IMC and compartmentalize their micronemes during their transformation into trophozoites. Sporozoites that failed to undergo conversion eventually died as noticed by extensive parasite vacuolization and degeneration of the nucleus at the ultrastructural level. We show that the most favorable environment for sporozoite conversion is the PV. This compartment may secure the parasite from hostile conditions and/or provide the host hepatic factors required for rapid transformation. To this point, it has been reported that shortly after invasion, intravacuolar parasites can upregulate the expression of host genes governing metabolite anabolism and transport [14]. To some extent, culture medium and host nucleoplasm can support parasite metamorphosis, but the speed and success rate of trophozoite development in these nonphysiological environments are much lower than in an intravacuolar niche $[12,27]$. Sparse information exists on the physiology of the PV or on the composition of its membrane in liver forms. The PV is permeable to small soluble molecules [15] and forms long extensions into the host cytoplasm [33]. During merozoite formation, the permeability of the PV increases considerably before this membrane finally ruptures, liberating parasites into the host cytoplasm [16]. So far, three proteins have been characterized and localized to the hepatic stage PVM: Exp-1 [34], UIS3 and UIS4 [33]. Interference with their expression impairs parasite development. The host Lfatty acid binding protein is docked to parasite UIS3 and may provide exogenous fatty acids to the parasite [35]. Host cholesterol scavenged by the parasite is also associated with the PVM [15]. Cholesterol depletion from host hepatocytes reduces the fluidity and permeability of 
this membrane [36]. Finally, another feature of the PVM is its intimate association with the host ER, which may represent a strategy developed by the parasite to retrieve nutrients for this organelle.

Micronemal proteins that promote hepatocyte invasion, such as TRAP, are not expressed anymore by liver forms $[37,38]$. We demonstrate that the TRAP-associated organelles are eliminated from the parasite during conversion. At the end of schizogony, newly formed micronemes contain specific proteins, e.g. AMA-1 [39], that will facilitate red blood cell invasion. Three biological systems implicated in organelle breakdown have been described in mammalian cells and yeast. First, the ubiquitin-proteasome system allows selective degradation of outlived organelles by the proteasome or lysosomes [40]. Second, autophagy is responsible for the bulk degradation of obsolete organelles via their enclosure within an autophagosome prior to fusion with lysosomes [18, 41, 42]. Third, to discard organelles, mammalian cells can use an alternative strategy that bypasses lysosomal digestion. In this case, the unwanted organelles are either enclosed in vacuoles that fuse with the plasma membrane and are therefore exocytosed or sequestered by the plasma membrane and pinch away from the cell resulting in expulsion [17, 43, 44]. Interestingly, evidence points to a potential role for each of these degradation processes during sporozoite conversion. For example, it has been demonstrated that proteasome inhibitors halt Plasmodium development in hepatocytes $[45,46]$. In addition, Plasmodium species contain a rudimentary set of autophagy genes [47], and autophagy is upregulated in blood stage parasites adapting to non-optimal conditions such as compromised vacuolar enzyme function [48]. Finally, the presence of membrane whorls within the PV of intrahepatic $P$. berghei suggests the activity of exocytosis or expulsion mechanisms [9].

During differentiation, reticulocytes expel their nucleus into the environment [17]. During this process, the nucleus moves to one side of the cell and then protrudes from the cell. Surrounded by little cytoplasm and an intact plasma membrane, the nucleus is subsequently appended to the cell by a thin pseudopodium-like membrane extension before complete detachment from the cell. Similar to the process of nucleus expulsion from reticulocytes, we observed whorls of IMC appended to the external surface of the cell by the parasite's plasma membrane, indicating that this organelle is extruded from converting parasites. We provide evidence that these packaged membranes consisting of IMC fragments are first detached from the inner face of the plasma membrane, and then tightly coiled inside the cytoplasm. Some IMC whorls surrounded by the plasma membrane are then expelled 'en bloc' by the parasite in a way resembling enucleation of maturing erythrocytes. The bulk expulsion of the IMC likely allows the parasite to attain its round shape. Of interest, a link between changes in IMC organization and the course of replication of the related parasite Toxoplasma has been recently reported [22]. The loss of IMC rigidity (by ablation of Rab11A associated with the IMC) in Toxoplasma results in incomplete cytokinesis, transforming the mother parasite into a syncytium instead of two individualized daughters.

The striking compaction and circular organization of the IMC, as seen in cross-section, is reminiscent of the multilamellae of myelin, in which the close packing of the sheaths is mediated by several integral membrane proteins $[49,50]$. The restructuring of the IMC in the parasite cytoplasm likely implies its dissociation from both actin filaments below the membrane bilayer and microtubules attached to the cytoplasmic side of the IMC [3]. Although the protein and lipid composition of the IMC remains largely unknown, we can assume that the packaging of the IMC into membranous whorls may be mediated by the cytoplasmic domain of proteins anchored in this organelle. Exocytosis of membranous structures, organized as lamellar arrays has also been observed in various tissues, such as in alveolar epithelial cells of the lungs [51] or in Schwalbe cells in the eye [52]. For these cells, expulsion of phospholipid-rich arrays has the common physiological function of decreasing the surface tension. In liver cells, extracellular evacuation of multilamellar arrays is frequent and associated with high autophagic activity [53]. Another example of exocytosis of myelin-like structures occurs in adipose tissue and is likely due to lipolytic activity in adipocytes [54]. Finally, retinal pigment epithelial cells secrete many residual bodies in the intercellular lateral space. These residual bodies consist of concentric membrane material, and are believed to be the end products of hydrolytic degradation [55].

Large cytosolic compartments containing micronemes may also be targeted to the exocytic pathway in metamorphosing sporozoites. The close apposition of these structures to the plasma membrane suggests eventual membrane fusion events, followed by the elimination of vesicle contents from the parasite. This hypothesis is compatible with our observations illustrating that TRAP-containing organelles are compartmentalized and released into the PV space at $24-48 \mathrm{~h}$ p.i. Such exocytic events must occur after IMC dismantling, since these structures are connected to the plasma membrane in areas lacking IMC. The origin of the limiting membrane of these exosome-like structures is unknown, but the docking of these compartments onto the parasite plasma 
membrane must involve the presence of molecular components that facilitate their recognition and fusion with the plasma membrane. Organelle expulsion clearly represents a very efficient mechanism to rapidly dispose of huge amounts of unwanted structures by converting parasites. One physical limitation to this process would be the narrowness of the vacuolar space. However, the $\mathrm{PV}$ of Plasmodium abounds in proteases [56] that might be implicated in the subsequent degradation of the expelled organelles. The selective clearance of some sporozoite organelles suggests a high degree of recognition of organelles involving temporally and spatially regulated components present on organellar membranes and of sorting for packing into vesicles for extracellular release. These vesicular carriers must also contain specific receptors for interaction with protein motors of the cytoskeleton, e.g. microtubules, in order to properly direct their cargo to the site of elimination. To this point, the Plasmodium liver stage system provides a unique model for studying the mechanisms implicated in the recruitment and selective exocytosis of unwanted organelles.

\section{Materials and Methods}

\section{Reagents and antibodies}

All chemicals were obtained from Sigma Chem. Co. (St Louis, MO) unless indicated otherwise. Mito-Tracker Green FM and ERTracker Red (Bodipy-TR glibenclamide) were obtained from Molecular Probes (Eugene, OR). Antibodies used for immunofluorescence assays included rabbit anti-MTIP (diluted at 1:500) from Dr L Bergman (Drexel University), rabbit anti-TRAP (diluted at 1:300) from Dr U Frevert (New York University), mouse anti-Hsp70 (diluted at 1:400), mouse anti-CSP (diluted at 1:1 000) from Dr F Zavala (Johns Hopkins University), rabbit anti-ACP (diluted at 1:100) from Dr S Prigge (Johns Hopkins University) and rabbit anti-UIS4 (diluted at 1:250) from Dr S Kappe (Seattle Biomedical Research Institute).

\section{Mammalian cell lines and parasite strains}

The commercial mammalian cell lines used in this study are the mouse Hepa 1-6 cells (ATCC CRL-1830) and the human HepG2-A16 cells (ATCC HB 8065). The HepG2 cell line stably transfected with CD81 (HepG2-CD81) was kindly provided by Dr D Mazier (Université Pierre et Marie Curie, France) [27]. All cell lines were grown as monolayers at $37{ }^{\circ} \mathrm{C}$ in an atmosphere of $5 \%$ $\mathrm{CO}_{2}$ in $\alpha$-minimum essential medium (Gibco BRL, Gaithersburg, MD), supplemented with $10 \%(\mathrm{v} / \mathrm{v})$ of fetal bovine serum (FBS), $2 \mathrm{mM}$ L-glutamine and penicillin/streptomycin (100 units/ml per $100 \mu \mathrm{g} / \mathrm{ml}$ ). The $P$. berghei ANKA line expressing GFP [57] was grown in Anopheles stephensi mosquitoes blood-fed on infected Swiss CD-1/ICR mice as described [12]. P. yoelii strain $17 \mathrm{XNL}$ was similarly grown in $A$. stephensi mosquitoes blood-fed on infected Swiss Webster mice.

\section{Plasmodium cultivation and infection in vitro}

$P$. berghei sporozoites were isolated from disrupted salivary glands of infected A. stephensi mosquitoes and further purified as described [12] before incubation in the presence of different mammalian cells for various times. Alternatively, sets of experiments were carried out on sporozoites maintained as axenic cultures for various times at $37{ }^{\circ} \mathrm{C}$ in DMEM containing $4.5 \mathrm{~g} / 1$ glucose and supplemented with L-glutamine to a final concentration of $2 \mathrm{mM}$, FBS at a final concentration of $10 \%$, penicillin-streptomycin at $500 \mathrm{U} / \mathrm{ml}$ and $500 \mu \mathrm{g} / \mathrm{ml}$, respectively, and Amphotericin B at 25 $\mu \mathrm{g} / \mathrm{ml}$.

\section{Light microscopy}

Immunofluorescence assays on intracellular parasites were performed as previously described [15]. Infected cells were incubated with the organelle-Trackers according to the manufacturer's instructions. Images were acquired on a Nikon Eclipse E800 microscope equipped with a Spot RT CCD Camera and processed using the Image-Pro Plus software (Media Cybernetics, Silver Spring, MD) and Adobe Photoshop software (Adobe Systems Inc.). To visualize the IMC in 3-D, intracellular $P$. berghe $i$ were labeled with anti-MTIP antibodies and fluorescence pattern was analyzed by a spinning disk confocal microscope (Nikon TE2000E) using a Plan-Apochromat $63 \times / 1.4 \mathrm{NA}$, and processed using the Volocity software (Improvison, Waltham, MA).

\section{Electron microscopy}

For thin-section transmission EM, pellets of $P$. berghei sporozoites, axenic parasites (P. berghei or P. yoelii) or P. yoelii-infected HepG2-CD81 were fixed in $2.5 \%$ glutaraldehyde (Electron Microscopy Sciences; EMS, Hatfield, PA) in $0.1 \mathrm{M}$ sodium cacodylate buffer $(\mathrm{pH} \mathrm{7.4)}$ for $1 \mathrm{~h}$ at room temperature, and processed as described [58] before examination with a Philips CM120 Electron Microscope (Eindhoven, The Netherlands) under $80 \mathrm{kV}$. For scanning EM imaging, preparations of axenic $P$. yoelii were washed in PBS, allowed to adhere on to poly(L-lysine)-coated glass coverslips and subsequently fixed in $2.5 \%$ glutaraldehyde in $0.1 \mathrm{M}$ cacodylate buffer ( $\mathrm{pH}$ 7.2). After fixation, samples were rinsed in $0.1 \mathrm{M}$ cacodylate buffer for $30 \mathrm{~min}$ at $4{ }^{\circ} \mathrm{C}$. They were then postfixed for $1 \mathrm{~h}$ in $2 \%$ osmium tetroxide at $4{ }^{\circ} \mathrm{C}$. After a brief rinse in distilled water, samples were en-bloc stained in $2 \%$ aqueous uranyl acetate for $1 \mathrm{~h}$ at room temperature in the dark. Following a graded ethanol dehydration, cells were dried to the critical point with liquid $\mathrm{CO}_{2}$, mounted onto SEM stubs with a double-stick carbon tape, and sputter coated with $10 \mathrm{~nm}$ of gold palladium. Samples were viewed and photographed with a LEO FESEM 1530 operating at $1 \mathrm{kV}$. For immunoelectron microscopy, axenic $P$. yoelii were fixed in 4\% paraformaldehyde (EMS) in 0.25 M HEPES (pH 7.4) for $1 \mathrm{~h}$ at room temperature, then in $8 \%$ paraformaldehyde in the same buffer overnight at $4{ }^{\circ} \mathrm{C}$. They were infiltrated, frozen and sectioned as previously described [58]. The sections were immunolabeled with anti-TRAP antibodies (1:50) or anti-MTIP antibodies (1:100), then with mouse anti-rabbit IgG antibodies, followed directly by $10 \mathrm{~nm}$ protein A-gold particles (Department of Cell Biology, Medical School, Utrecht University, The Netherlands).

\section{Acknowledgments}

The authors are grateful to all members of the Carruthers and Coppens laboratories for their helpful discussions during the course of this work. We wish to thank Dabeiba Bernal-Rubio, San- 
dra Gonzalez and Jean Nonan from New York University School of Medicine for their remarkable technical support in the rearing and infection of the mosquitoes. We are grateful to the generous providers of antibodies against Plasmodium antigens. We would also like to thank the competent technical staff from the Microscopy Facility at Johns Hopkins University and Yale University. This research was supported by the Johns Hopkins Malaria Research Institute and the Bloomberg Family Foundation. BJ was a recipient of a predoctoral MRI fellowship.

\section{References}

1 Prudencio M, Rodriguez A, Mota MM. The silent path to thousands of merozoites: the Plasmodium liver stage. Nat Rev Microbiol 2006; 4:849-856.

2 Sturm A, Amino R, van de Sand C, et al. Manipulation of host hepatocytes by the malaria parasite for delivery into liver sinusoids. Science 2006; 313:1287-1290.

3 Morrissette NS, Sibley LD. Cytoskeleton of apicomplexan parasites. Microbiol Mol Biol Rev 2002; 66:21-38.

4 Kudryashev M, Lepper S, Stanway R, et al. Positioning of large organelles by a membrane-associated cytoskeleton in Plasmodium sporozoites. Cell Microbiol 2010; 12:362-371.

5 Robson KJ, Frevert U, Reckmann I, et al. Thrombospondinrelated adhesive protein (TRAP) of Plasmodium falciparum: expression during sporozoite ontogeny and binding to human hepatocytes. EMBO J 1995; 14:3883-3894.

6 Sinnis P, Sim BK. Cell invasion by the vertebrate stages of Plasmodium. Trends Microbiol 1997; 5:52-58.

7 Tufet-Bayona M, Janse CJ, Khan SM, Waters AP, Sinden RE, Franke-Fayard B. Localisation and timing of expression of putative Plasmodium berghei rhoptry proteins in merozoites and sporozoites. Mol Biochem Parasitol 2009; 166:22-31.

8 Meis JF, Verhave JP, Jap PH, Meuwissen JH. Transformation of sporozoites of Plasmodium berghei into exoerythrocytic forms in the liver of its mammalian host. Cell Tissue Res 1985; 241:353-360.

9 Meis JF, Verhave JP, Jap PH, Sinden RE, Meuwissen JH. Malaria parasites--discovery of the early liver form. Nature 1983; 302:424-426.

10 Meis JF, Verhave JP, Jap PH, Sinden RE, Meuwissen JH. Ultrastructural observations on the infection of rat liver by Plasmodium berghei sporozoites in vivo. J Protozool 1983; 30:361-366.

11 Frevert U. Sneaking in through the back entrance: the biology of malaria liver stages. Trends Parasitol 2004; 20:417-424.

12 Kaiser K, Camargo, Kappe SH. Transformation of sporozoites into early exoerythrocytic malaria parasites does not require host cells. J Exp Med 2003; 197:1045-1050.

13 Tarun AS, Peng X, Dumpit RF, et al. A combined transcriptome and proteome survey of malaria parasite liver stages. Proc Natl Acad Sci USA 2008; 105:305-310.

14 Singh AP, Buscaglia CA, Wang Q, et al. Plasmodium circumsporozoite protein promotes the development of the liver stages of the parasite. Cell 2007; 131:492-504.

15 Bano N, Romano JD, Jayabalasingham B, Coppens I. Cellular interactions of Plasmodium liver stage with its host mammalian cell. Int J Parasitol 2007; 37:1329-1341.

16 Sturm A, Graewe S, Franke-Fayard B, et al. Alteration of the parasite plasma membrane and the parasitophorous vacuole membrane during exo-erythrocytic development of malaria parasites. Protist 2009; 160:51-63.

17 Blanc L, De Gassart A, Geminard C, Bette-Bobillo P, Vidal M. Exosome release by reticulocytes--an integral part of the red blood cell differentiation system. Blood Cells Mol Dis 2005; 35:21-26.

18 Bowerman B, Kurz T. Degrade to create: developmental requirements for ubiquitin-mediated proteolysis during early $\mathrm{C}$. elegans embryogenesis. Development 2006; 133:773-784.

19 Kim J, Klionsky DJ. Autophagy, cytoplasm-to-vacuole targeting pathway, and pexophagy in yeast and mammalian cells. Annu Rev Biochem 2000; 69:303-342.

20 White NJ. The role of anti-malarial drugs in eliminating malaria. Malar J 2008; 7 Suppl 1:S8.

21 Bergman LW, Kaiser K, Fujioka H, et al. Myosin A tail domain interacting protein (MTIP) localizes to the inner membrane complex of Plasmodium sporozoites. J Cell Sci 2003; 116:39-49.

22 Agop-Nersesian C, Naissant B, Ben Rached F, et al. Rab11Acontrolled assembly of the inner membrane complex is required for completion of apicomplexan cytokinesis. PLoS Pathog 2009; 5:e1000270.

23 Tsuji M, Mattei D, Nussenzweig RS, Eichinger D, Zavala F. Demonstration of heat-shock protein 70 in the sporozoite stage of malaria parasites. Parasitol Res 1994; 80:16-21.

24 Puhka M, Vihinen H, Joensuu M, Jokitalo E. Endoplasmic reticulum remains continuous and undergoes sheet-to-tubule transformation during cell division in mammalian cells. $J$ Cell Biol 2007; 179:895-909.

25 Waller RF, McFadden GI. The apicoplast: a review of the derived plastid of apicomplexan parasites. Curr Issues Mol Biol 2005; 7:57-79.

26 Stanway RR, Witt T, Zobiak B, Aepfelbacher M, Heussler VT. GFP-targeting allows visualization of the apicoplast throughout the life cycle of live malaria parasites. Biol Cell 2009; 101:415-430.

27 Silvie O, Greco C, Franetich JF, et al. Expression of human CD81 differently affects host cell susceptibility to malaria sporozoites depending on the Plasmodium species. Cell Microbiol 2006; 8:1134-1146.

28 Cowan G, Krishna S, Crisanti, Robson K. Expression of thrombospondin-related anonymous protein in Plasmodium falciparum sporozoites. Lancet 2002; 339:1412-1413.

29 Rogers WO, Malik A, Mellouk S, et al. Characterization of Plasmodium falciparum sporozoite surface protein 2. Proc Natl Acad Sci USA 1992; 89:9176-9180.

30 Baton LA, Ranford-Cartwright LC. Spreading the seeds of million-murdering death: metamorphoses of malaria in the mosquito. Trends Parasitol 2005; 21:573-580.

31 Carter V, Nacer AM, Underhill A, Sinden RE, Hurd H. Minimum requirements for ookinete to oocyst transformation in Plasmodium. Int J Parasitol 2007; 7:1221-1232.

32 Khater EI, Sinden RE, Dessens JT. A malaria membrane skeletal protein is essential for normal morphogenesis, motility, and infectivity of sporozoites. J Cell Biol 2004; 167:425-432.

33 Mueller AK, Camargo N, Kaiser K, et al. Plasmodium liver stage developmental arrest by depletion of a protein at the parasite-host interface. Proc Natl Acad Sci USA 2005; 
102:3022-3027.

34 Simmons D, Woollett G, Bergin-Cartwright M, Kay D, Scaife $\mathrm{J}$. A malaria protein exported into a new compartment within the host erythrocyte. EMBO J 1978; 6:485-491.

35 Mikolajczak SA, Jacobs-Lorena V, MacKellar DC, Camargo N, Kappe SH. L-FABP is a critical host factor for successful malaria liver stage development. Int J Parasitol 2007; 37:483489.

36 Silvie O, Charrin S, Billard M, et al. Cholesterol contributes to the organization of tetraspanin-enriched microdomains and to CD81-dependent infection by malaria sporozoites. J Cell Sci 2006; 119:1992-2002.

37 Wang Q, Brown S, Roos DS, Nussenzweig V, Bhanot P. Transcriptome of axenic liver stages of Plasmodium yoelii. Mol Biochem Parasitol 2004; 137:161-168.

38 Amino R, Thiberge S, Martin B, et al. Quantitative imaging of Plasmodium transmission from mosquito to mammal. Nat Med 2006; 12:220-224.

39 Triglia T, Healer J, Caruana SR, et al. Apical membrane antigen 1 plays a central role in erythrocyte invasion by Plasmodium species. Mol Microbiol 2000; 38:706-718.

40 Hershko A, Ciechanover A. The ubiquitin system. Annu Rev Biochem 1998; 67:425-479.

41 Mizushima N. Autophagy: process and function. Genes Dev 2007; 21:2861-2873.

42 Ravikumar B, Futter M, Jahreiss L, et al. Mammalian macroautophagy at a glance. J Cell Sci 2009; 122:1707-1711.

43 Gronowicz G, Swift H, Steck TL. Maturation of the reticulocyte in vitro. J Cell Sci 1984; 71:177-197.

44 Hebiguchi M, Hirokawa M, Guo YM, et al. Dynamics of human erythroblast enucleation. Int J Hematol 2008; 88:498507.

45 Gantt SM, Myung JM, Briones MR, et al. Proteasome inhibitors block development of Plasmodium spp. Antimicrob Agents Chemother 1998; 42:2731-2738.

46 Lindenthal C, Weich N, Chia YS, Heussler V, Klinkert MQ. The proteasome inhibitor MLN-273 blocks exoerythrocytic and erythrocytic development of Plasmodium parasites. Parasitology 2005; 131:37-44.

47 Rigden DJ, Michels PA, Ginger ML. Autophagy in protists: examples of secondary loss, lineage-specific innovations, and the conundrum of remodeling a single mitochondrion. $A u$ tophagy 2009; 5:784-94.

48 Bonilla JA, Bonilla TD, Yowell CA, Fujioka H, Dame JB. Critical roles for the digestive vacuole plasmepsins of Plasmodium falciparum in vacuolar function. Mol Microbiol 2007; 65:64-75.

49 D’Urso D, Brophy PJ, Staugaitis SM, et al. Protein zero of peripheral nerve myelin: biosynthesis, membrane insertion, and evidence for homotypic interaction. Neuron 1990; 4:449-460.

50 Montague P, McCallion AS, Davies RW, Griffiths IR. Myelinassociated oligodendrocytic basic protein: a family of abundant CNS myelin proteins in search of a function. Dev Neurosci 2006; 28:479-487.

51 Kalina M, Pease DC. The preservation of ultrastructure in saturated phosphatidyl cholines by tannic acid in model systems and type II pneumocytes. J Cell Biol 1977; 74:726-741.

52 Raviola G. Schwalbe line's cells: a new cell type in the trabecular meshwork of Macaca mulatta. Invest Ophthalmol Vis Sci 1982; 22:45-56.

53 Kerr JF. Liver cell defaecation: an electron-microscope study of the discharge of lysosomal residual bodies into the intercellular space. J Pathol 1970; 100:99-103.

54 Amende LM, Blanchette-Mackie EJ, Scow RO. Demonstration of fatty acid domains in membranes produced by lipolysis in mouse adipose tissue. A freeze-fracture study. Cell Tissue Res 1986; 246:495-508.

55 Rungger-Brändle E, Englert U, Leuenberger PM. Exocytic clearing of degraded membrane material from pigment epithelial cells in frog retina. Invest Ophthalmol Vis Sci 1987; 28:2026-2037.

56 Nyalwidhe J, Lingelbach K. Proteases and chaperones are the most abundant proteins in the parasitophorous vacuole of Plasmodium falciparum-infected erythrocytes. Proteomics 2006; 6:1563-1573.

57 Franke-Fayard B, Trueman H, Ramesar J, et al. A Plasmodium berghei reference line that constitutively expresses GFP at a high level throughout the complete life cycle. Mol Biochem Parasitol 2004; 127:23-33.

58 Nishikawa Y, Quittnat F, Stedman TT, et al. Host cell lipids control cholesteryl ester synthesis and storage in intracellular Toxoplasma. Cell Microbiol 2005; 7:849-867.

Supplementary information is linked to the online version of the paper on the Cell Research website.) 